\title{
Sliding Mode Active Vibration Control and Three Axis Attitude Control using Four Reaction Wheels
}

\author{
Farhad Fani Saberi \\ Amirkabir University of \\ Technology \\ Space Science and \\ Technology Institute \\ Tehran, Iran
}

\author{
Soheil Khalajian \\ Amirkabir University of \\ Technology \\ Aerospace Engineering \\ Department \\ Tehran, Iran
}

\author{
Mahmoud Mani \\ Amirkabir University of \\ Technology \\ Aerospace Engineering \\ Department \\ Tehran, Iran
}

\begin{abstract}
The most important problems in satellites are their energy supply and decreasing weight. One of the solutions is using flexible appendages. Flexibility can supply both energy and weight loss in satellites. Also other appendages like antennas and mechanical arms can be flexible. But flexibility can produce some vibrations. These vibrations cause complexity attitude control systems due to flexible appendages.

In this paper, the attitude control of flexible satellite by using four reaction wheels in presence of gravity gradient and orbit frequency has been considered. The dynamic of flexible appendages have been derived from energy equations and Lagrange method. Also, momentum management and minimization of the momentum have been employed to avoid of reaction wheels saturation. Then, the control command law using the quaternion error vector for attitude control of rigid body and sliding mode control (SMC) for active suppuration of flexible appendages have been used. Finally the performance of control system between presence and absence of reaction wheels has been compared.
\end{abstract}

\section{Keywords}

Flexible satellite, Three-axis attitude control, Sliding mode control, Reaction wheel, Momentum management

\section{INTRODUCTION}

A typical satellite consists of two principal parts. The first one is rigid body of the satellite and the second are some flexible appendages. Therefore, in order to control a flexible satellite, vibrations due to the flexible appendages have been considered. In [1], a spinning maneuver and vibrations of a flexible satellite in orbit has been controlled using an active controller. The maneuver has been assumed only around one axis. In [2], a notch filter has been used to reduce vibration of appendages. In this reference, designing notch filter method based on adaptive control in orbit technology considering frequency potential of vibrations has been proposed. Also, a controller just in one channel has been employed for an example.

An attitude control of satellite with considering fast maneuvers and saturation constraint has been presented in [3], but reaction wheels and their effects on control performance have not been considered in this reference. In [4], attitude control of a satellite with imagery mission by three-axis stabilization zero momentum method has been discussed. They have supplied PID controller.

In [5], modeling uncertainty in elastic structure has been expressed. In this work the expression of uncertainty in natural frequency and damping vibrational mode has been intended. Also, satellite normalization equation of motion for design controller and attitude control of elastic satellite has been expressed by the second theorem of Lyapunov. In [6], attitude of a satellite with solar panels by adaptive fuzzy controller has been studied. The performance of the system has been improved and the control torque has been reduced, but resulting equations are complex. In [7], adaptive fault tolerant control of flexible satellite based on SDU factoring without error detection has been designed. The advantage of this method is ensuring stability and asymptotic tracking.

One of the problems in control design is uncertainty. The model uncertainty may be occurred due to the plan uncertainty or choosing simplified view of the dynamic system. From the perspective of control, it can divide the model uncertainty to two main types of structure uncertainties (or parametric) and non-structure uncertainties (or unmodeled dynamics) classification. Also, the other important issue is control robustness against external disturbance. So, using robust control in vibrations control is very important.

A simple method for robust control is sliding mode controller. Sliding mode is a regulated method for stabilization problem and consistence performance in face of modeling inaccuracies [8] and also external disturbances.

In this paper, a control law includes two loops for a flexible satellite in a circular orbit around the earth and under effect of gravity gradient has been designed. The first loop is a control command law based on quaternion error vector for three-axis attitude control of flexible satellite rigid part. In this case, control torques have been tried to be minimum during an acceptable maneuver and given the large size of the satellite, by selecting appropriate control gain coefficients. Also, four reaction wheels have been considered as actuators. Because, the efficiency and reliability of system has been increased by using four wheels when one of the wheels get damaged. To avoid of reaction wheels saturation, momentum management and minimization of the momentum have been employed.

Also in second loop a sliding mode control (SMC) for damping vibrations of flexible part has been designed. Simulations show the sliding mode control is working properly in the presence of uncertainty and external disturbances effects of reaction wheels on the flexible appendages. Finally the performance of control system has been compared between the presence and the absence of reaction wheels.

This paper is organized as follows. Section 2 presents the mathematical model of flexible satellite. Section 3 describes the control command law using the quaternion error vector and their equations. Section 4 presents the sliding mode 
control for damping vibrations of flexible appendages. Section 5 presents the simulation and curves. In section 6 , the conclusions of study have been represented.

\section{MATHEMATICAL MODEL OF FLEXIBLE SATELLITE}

A flexible satellite is composed of a rigid main body and some flexible appendages. The kinematics of the satellite determines the attitude of the main body and are described by the four unitary quaternion [9]:

$q=e \sin \left(\frac{\beta}{2}\right), \cos \left(\frac{\beta}{2}\right)$

where $e \in R^{3}$ and $\beta$ denote the Euler axis and Euler angle, respectively. The quaternion vector is considered $\bar{q}=\left[\begin{array}{ll}q_{0} & q^{T}\end{array}\right]_{\text {with }}^{T} q \in R^{3}$. Then the kinematic equations are described in terms of the attitude quaternion and are given by following equation:

$\dot{\bar{q}}=\frac{1}{2}\left[\begin{array}{l}-q^{T} \\ {[q \times]+q_{0} I_{3 \times 3}}\end{array}\right] \omega$

Where $\omega \in R^{3}$ denote the angular velocity vector and $I_{3 \times 3}$ is the $3 \times 3$ identity matrix. The skew-symmetric matrix $[q \times]$ is:

$[q \times]=\left[\begin{array}{ccc}0 & -q_{3} & q_{2} \\ q_{3} & 0 & -q_{1} \\ -q_{2} & q_{1} & 0\end{array}\right]$

and the elements of $q$ are restricted by $\|\bar{q}\|=1$. Note that a quaternion consists of the scalar $q_{0}$ and the three dimensional vector $^{q}$, so it has four components. The scalar term is used for avoidance of singular points in the attitude representation. The quaternion kinematics equation is required to be solved for all four components. However, to indicate the orientation of the satellite or a rotational motion, it is sufficient to use only the vector $q$ because this vector completely shows rotation axis and angle. Furthermore, the scalar $q_{0}$ can be calculated easily using the vector $q$ and the condition $\|\bar{q}\|=1$. More details of quaternion and other attitude representations can be found in $[9,10]$.

The equation governing a flexible satellite is expressed as [11]:

$$
\begin{aligned}
& J \dot{\omega}+\delta^{T} \ddot{\eta}=-[\omega \times]\left(J \omega+\delta^{T} \dot{\eta}\right)+u+d \\
& \ddot{\eta}+C \dot{\eta}+K \eta=-\delta \dot{\omega}+u_{f}
\end{aligned}
$$

where $J=J^{T}$ is the total inertia matrix of the satellite, $\eta$ is the modal displacement, and $\delta$ is the coupling matrix between the central rigid body and the flexible attachments. $u \in R^{3}$ denotes the control input, $d \in R^{3}$ represents the external disturbance torque, and $K$ and $C$ denote the stiffness and damping matrices, respectively, which are defined as

$$
\begin{aligned}
& K=\operatorname{diag}\left(\omega_{n i}^{2}, i=1,2, \ldots, N\right) \\
& C=\operatorname{diag}\left(2 \xi_{i} \omega_{n i}, i=1,2, \ldots, N\right)
\end{aligned}
$$

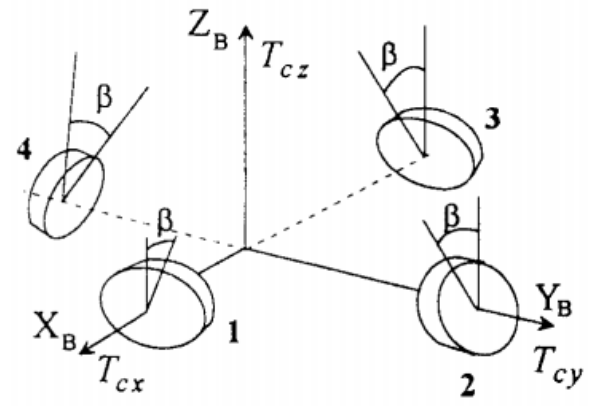

Fig 1: Four reaction wheels

In this section, one possible geometrical configuration of a control system based on four reaction wheels has been analyzed; see Figure (1) the rotational axes of the four wheels are inclined to the $X_{B}-Y_{B}$ plane by an angle $\beta$. Because of this inclination, each wheel can apply torques and momentum in [12] the $Z_{B}$ direction also. The torques produced along the three body axes are $\hat{T}_{c x}, \hat{T}_{c y}, \hat{T}_{c z}$. Thus it has the following relations:

$$
\begin{aligned}
& {\left[\begin{array}{l}
T_{1} \\
T_{2} \\
T_{3} \\
T_{4}
\end{array}\right]=\frac{1}{2}\left[\begin{array}{cccc}
1 & 0 & \frac{1}{2} & \frac{1}{2} \\
0 & 1 & \frac{1}{2} & -\frac{1}{2} \\
-1 & 0 & \frac{1}{2} & \frac{1}{2} \\
0 & -1 & \frac{1}{2} & -\frac{1}{2}
\end{array}\right]\left[\begin{array}{l}
\hat{T}_{c x} \\
\hat{T_{c y}} \\
\hat{T_{c z}} \\
0
\end{array}\right]} \\
& {\left[\begin{array}{l}
\hat{T_{c x}} \\
\hat{T_{c y}} \\
\hat{T_{c z}}
\end{array}\right]=\left[\begin{array}{l}
T_{c x} / \cos \beta \\
T_{c y} / \cos \beta \\
T_{c z} / \sin \beta
\end{array}\right]}
\end{aligned}
$$

Where $T=\left[\begin{array}{llll}T_{1} & T_{2} & T_{3} & T_{4}\end{array}\right]^{T}$ is the control torque form four reaction wheel, $T$ can be minimized. So Hamiltonian $H$ is [12]:

$H=\sum_{i=1}^{4} T_{i}^{2}$

That finally will be minimize.

Also, momentum minimization $\left|h_{w}\right|=\sum h_{w i}^{2}$ must be employ.

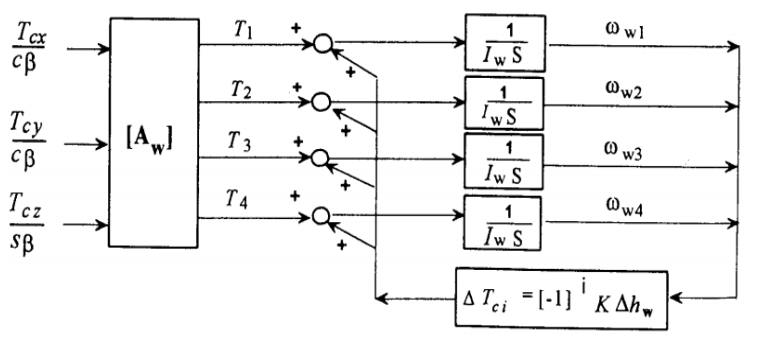

Fig 2: If momentum management control of four wheels

The attitude of satellite can be controlled by momentum angular commands instead of attitude control commands with control torques. Here momentum vector $h_{w i}=I_{w i} \omega_{w i}$ should be minimized instead of control torque vector. Minimization of momentum vector satisfy following condition: 
$\Delta h_{w}=h_{w 1}-h_{w 1}+h_{w 3}-h_{w 4}=0$

And finally:

$\Delta T_{c i}=[-1] K \Delta h_{w}, \quad i=1, \ldots, 4$

That causes become a momentum management feedback loop. You can see [12].

For employ the orbit frequency following matrix can be define [12]:

$\left[\begin{array}{l}\dot{q}_{1} \\ \dot{q}_{2} \\ \dot{q}_{3} \\ \dot{q}_{4}\end{array}\right]=\frac{1}{2}\left[\begin{array}{cccc}0 & \omega_{3} & -\omega_{2}+n & \omega_{1} \\ -\omega_{3} & 0 & \omega_{1} & \omega_{2}+n \\ \omega_{2}-n & -\omega_{1} & 0 & \omega_{3} \\ -\omega_{1} & -\omega_{2}+n & -\omega_{3} & 0\end{array}\right]\left[\begin{array}{l}q_{1} \\ q_{2} \\ q_{3} \\ q_{4}\end{array}\right]$

Where $n$ is orbital period. The gravity gradient vector $G=\left[\begin{array}{lll}G_{x} & G_{y} & G_{z}\end{array}\right]^{T}$ is defined by [12]:

$G=\frac{3 \mu}{2 R_{0}^{5}} \int_{M}[R \bullet \rho \amalg \rho \times R] d m$

\section{CONTROL COMMAND LAW USING THE QUATERNION ERROR VECTOR}

There exists an equivalent quaternion error vector that expresses the attitude error between (i) the satellite attitude direction in space and (ii) the target direction toward which the satellite is oriented at the end of the attitude maneuver [12]. for derive the quaternion error vector it can be written as follows:

$\left[A\left(q_{E}\right)\right]=\left[A\left(q_{T}\right)\right]\left[A\left(q_{S}\right)\right]^{-1}=\left[A\left(q_{T}\right)\right]\left[A\left(q_{S}^{-1}\right)\right]$

In quaternion notation, above equation leads to:

$q_{S}^{-1} q_{T}=q_{E}=\left[\begin{array}{cccc}q_{T 4} & q_{T 3} & -q_{T 2} & q_{T 1} \\ -q_{T 3} & q_{T 4} & q_{T 1} & q_{T 2} \\ q_{T 2} & -q_{T 1} & q_{T 4} & q_{T 3} \\ -q_{T 1} & -q_{T 2} & -q_{T 3} & q_{T 4}\end{array}\right]\left[\begin{array}{c}-q_{S 1} \\ -q_{S 2} \\ -q_{S 3} \\ q_{S 1}\end{array}\right]$

where $q_{S}, q_{T}$, and $q_{E}$ are (respectively) the error, target, and satellite quaternions. So, this result the following control vector [9]:

$T_{c x}=2 K_{x} q_{1 E} q_{4 E}+K_{x d} p$

$T_{c y}=2 K_{y} q_{2 E} q_{4 E}+K_{y d} q$

$T_{c z}=2 K_{z} q_{3 E} q_{4 E}+K_{z d} r$

the satellite quaternion vector is obtained by integrating equation (11). Only three elements need to be integratednamely, $q_{S 1}, q_{S 2}$, and $q_{S 1}$ since $q_{S 4}$ is known from the relation $|q|=1$. Performing the computation of $q_{E}$ in equation (14) requires fewer algebraic operations than computing the elements in $\left[A_{E}\right]$.

\section{SLIDING MODE CONTROL}

Here, sliding mode control for active vibration suppuration of flexible appendages of satellite has been design, and the end has been compared with PD controller.

Flexible part of equation (4) in state space is:

$\dot{X}=A X+u$

Where:
$X=\left[\begin{array}{lllll}\eta_{1} & \dot{\eta}_{1} & \cdots & \eta_{N} & \dot{\eta}_{N}\end{array}\right] ; \quad A=\left[\begin{array}{cccccc}0 & 1 & 0 & 0 & \cdots & 0 \\ -\omega_{1}^{2} & -2 \xi_{1} \omega_{1} & 0 & 0 & \cdots & 0 \\ 0 & 0 & 0 & 1 & \cdots & 0 \\ 0 & 0 & -\omega_{2}^{2} & -2 \xi_{2} \omega_{2} & \cdots & 0 \\ \vdots & \vdots & \vdots & \vdots & \ddots & \vdots \\ 0 & 0 & 0 & 0 & -\omega_{N}^{2} & -2 \xi_{N} \omega_{N}\end{array}\right]$

Where $N$ is the number of vibrational modes.

In vibrations, the first multi-mode is dominant modes and have a greater effect on the dynamics of the system. In this paper damping the first three modes have been considered. Also the presented algebra for more and less modes has been extended. So it can be written as follows:

$\left\{\begin{array}{l}\dot{x}_{1}=\dot{x}_{2} \\ \dot{x}_{2}=f_{1}\left(x_{1}\right)+b_{1} u \\ \vdots \\ \dot{x}_{n-1}=\dot{x}_{n} \\ \dot{x}_{n}=f_{n-1}\left(x_{n-1}\right)+b_{n-1} u\end{array}\right.$

Tracking error in value $x$ as $\tilde{x}=x-x_{d}$ has been assumed, and sliding surface define by [13]:

$s(x, t)=\left[\frac{d}{d t}+\lambda\right]^{(n-1)} \tilde{x}$

Where $n$ is order of system. Then:

$s(x, t)=\dot{\tilde{x}}+\lambda \tilde{x}$

$u_{e q}$ is obtained by equating $\dot{s}$ with zero, and with considering $i$ modes can be written as following:

$u_{\text {eqi }}=-\left(\lambda_{i} x_{2 i}+g_{i}\right) / b_{i}$

Finally, to Resistant against disturbances and avoiding of chattering and also guarantee the stability of system a discontinues part to controller can be added as following:

$u=u_{\text {eqi }}-K \operatorname{sign}(s)$

Where $K$ is positive.

\section{SIMULATION}

An example for a flexible satellite in the orbit $h=800$ and $\omega_{0}=0.00104$ has been considered to verifying. The amount of parameters of dynamic and control have been specified in following tables.

Table 1. The parameter of dynamic of flexible satellite

\begin{tabular}{|c|c|c|c|c|}
\hline $\begin{array}{l}\text { Param } \\
\text { eter }\end{array}$ & $\begin{array}{c}\text { Param } \\
\text { eter } \\
\text { name }\end{array}$ & \multicolumn{3}{|c|}{ Amount } \\
\hline \multicolumn{5}{|c|}{ Rigid body } \\
\hline$J$ & $\begin{array}{c}\text { Moment of } \\
\text { inertia }\end{array}$ & {$\left[\begin{array}{c}350 \\
3 \\
4\end{array}\right.$} & $\left.\begin{array}{cc}3 & 4 \\
270 & 10 \\
10 & 190\end{array}\right]$ & $k g . m^{2}$ \\
\hline$\delta$ & $\begin{array}{l}\text { Coupling } \\
\text { matrix }\end{array}$ & {$\left[\begin{array}{c}6.45637 \\
-1.25619 \\
1.11678 \\
1.23637\end{array}\right.$} & $\begin{array}{cc}1.27814 & 2.15629 \\
0.91756 & -1.67264 \\
2.48901 & -0.83674 \\
-2.6581 & -1.12503\end{array}$ & $\mathrm{~kg}^{1 / 2} \cdot \mathrm{m} / \mathrm{s}^{2}$ \\
\hline$I_{w}$ & $\begin{array}{c}\text { Moment of } \\
\text { inertia of } \\
\text { wheels }\end{array}$ & & $0.1 \mathrm{~kg} . \mathrm{m}^{2}$ & \\
\hline$k_{w}$ & $\begin{array}{c}\text { Coefficient } \\
\text { of } \\
\text { momentum } \\
\text { inertia }\end{array}$ & & 0.2 & \\
\hline - & $\begin{array}{c}\text { Maximum } \\
\text { wheel } \\
\text { torque }\end{array}$ & & $0.5 \mathrm{Nm}$ & \\
\hline
\end{tabular}




\begin{tabular}{|c|c|c|}
\hline \multicolumn{3}{|c|}{ Flexible appendages } \\
\hline$\omega_{1}$ & $\begin{array}{c}\text { First } \\
\text { natural } \\
\text { frequency }\end{array}$ & $0.7481 \mathrm{rad} / \mathrm{s}$ \\
\hline$\omega_{2}$ & $\begin{array}{c}\text { Second } \\
\text { natural } \\
\text { frequency }\end{array}$ \\
$\omega_{3}$ & $\begin{array}{c}\text { Third } \\
\text { natural } \\
\text { frequency }\end{array}$ & $1.1038 \mathrm{rad} / \mathrm{s}$ \\
\hline$\xi_{1}$ & $\begin{array}{c}\text { First } \\
\text { damping } \\
\text { coefficient }\end{array}$ & $1.8733 \mathrm{rad} / \mathrm{s}$ \\
\hline$\xi_{2}$ & $\begin{array}{c}\text { Second } \\
\text { damping } \\
\text { coefficient }\end{array}$ & 0.0056 \\
\hline$\xi_{3}$ & $\begin{array}{c}\text { Third } \\
\text { damping } \\
\text { coefficient }\end{array}$ & 0.0086 \\
\hline
\end{tabular}

At the first, assume that all initial angular velocity and Euler angles are equal to zero. During the maneuver, the satellite wants to take the position $\left[\begin{array}{lll}\phi & \theta & \psi\end{array}\right]=\left[\begin{array}{lll}-5 & 2 & 1\end{array}\right](\mathrm{deg})$.

Simulations show that the performance of control system in presence of external disturbance and uncertainty and also reaction wheels disturbance is satisfying. As shown in figure 3 , the modal displacements have been damped to zero appropriately. Also, damping displacements in absence of actuator are smoothly in compared with presence of actuator. Also the settling time in absence of actuator is slightly less. This roughness and time delays are occurring due to reaction wheels disturbance in the flexible satellite. The effect of actuator in this figure is clearly. Also, sliding mode has appropriate performance despite of the disturbance and uncertainty effects on the vibrations of flexible appendages.

Table 2. The coefficient gain of control

\begin{tabular}{|c|c|c|c|}
\hline Parameter & Amount & Parameter & Amount \\
\hline \multicolumn{3}{|c|}{$\begin{array}{c}\text { Coefficients of the control command low using the } \\
\text { quaternion error vector }\end{array}$} \\
\hline$K_{x}$ & 30 & $K_{d x}$ & 50 \\
\hline$K_{y}$ & 20 & $K_{d y}$ & 30 \\
\hline
\end{tabular}

\begin{tabular}{|c|c|c|c|}
\hline$K_{z}$ & 18 & $K_{d z}$ & 34 \\
\hline \multicolumn{3}{|c|}{ Coefficients of PD for flexible appendages } \\
\hline$K_{p}$ & 16 & $K_{d}$ & 10 \\
\hline \multicolumn{4}{|c|}{ Coefficients of sliding mode } \\
\hline$K$ & 16 & $\lambda_{2}$ & 5 \\
\hline$\lambda_{1}$ & 5 & $\lambda_{3}$ & 5 \\
\hline
\end{tabular}

As shown in figure 4, Euler angles have been shown. Presence of actuator has been caused increasing domain and also decreasing settling time in the pitch and yaw angles. But the roll has been decreased in presence of actuator. Also, attitude actuator has been caused time delay in the maneuvers. Exist of physical constraints and saturation levels (maximum torque of wheels) limit the ability to generate torque. They are agents of the time delay.

Figure 5 shows angular velocities. The time delay also is clear. Specially, domain of $\omega_{3}$ curve has been increased because presence of the attitude actuator (reaction wheels). Also, $\omega_{2}$ has not been achieved to zero (exist of steady state error) because orbital frequency.

As shown in figure 6 and figure 7, the time delays and domain changes have been represented clearly. In figure 6 , the settling time of control torque has been increased because the presence of attitude actuator. Also, domain of $T_{c 3}$ has been increased. In figure 7 , vibrations of flexible appendage curve is not smoothly due to effects of reaction wheels.

Figure 8 shows the control torque of attitude actuator. Figure 9 shows the angular velocities of four reaction wheels with momentum management feedback. The angular velocities have not been achieved to zero due to presence of disturbance an also orbital frequency. This figure also shows the angular momentum contribution of each wheel. In figure 10 , $\Delta h_{w}=h_{w 1}-h_{w 1}+h_{w 3}-h_{w 4}=0$ condition with momentum feedback loop that cause minimizations $H=\sum_{i=1}^{4} T_{i}^{2}$ and $\left|h_{w}\right|=\sum h_{w i}^{2}$ has been shown. 

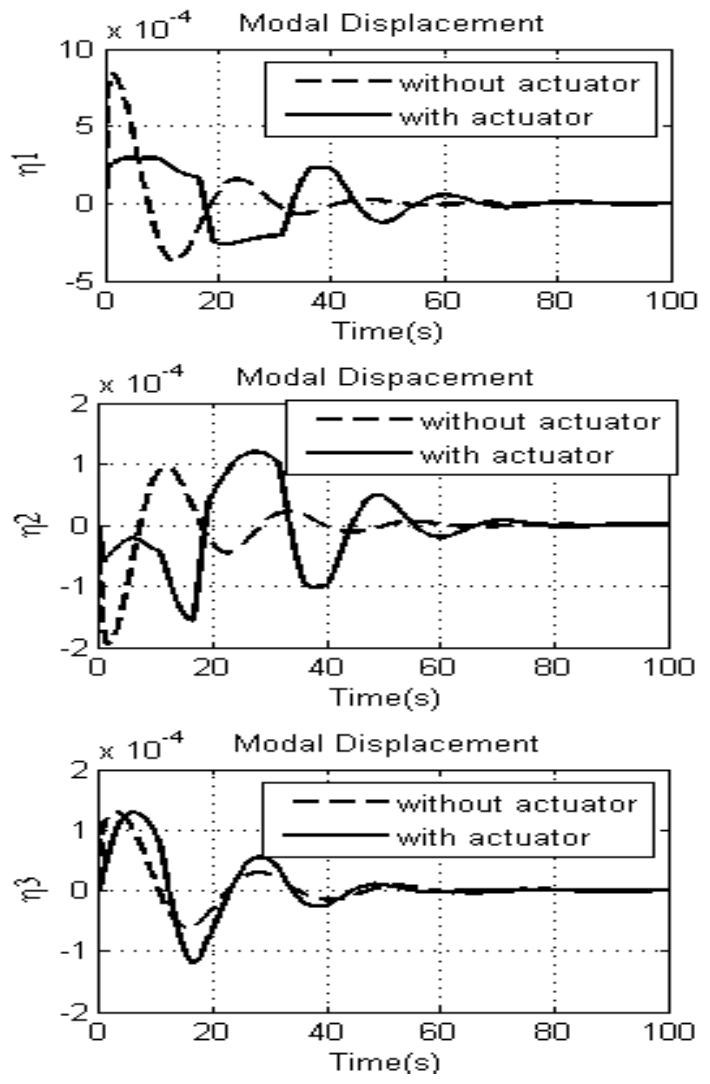

Fig 3: Modal displacement in presence of disturbance
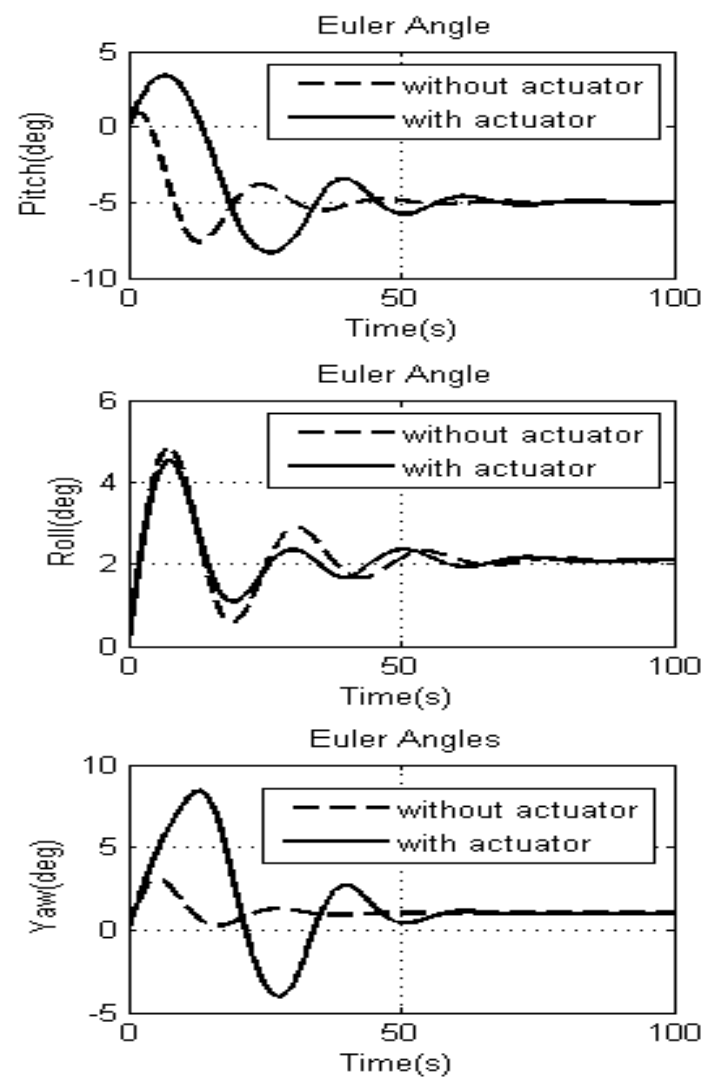

Fig 4: Euler angle in presence of disturbance
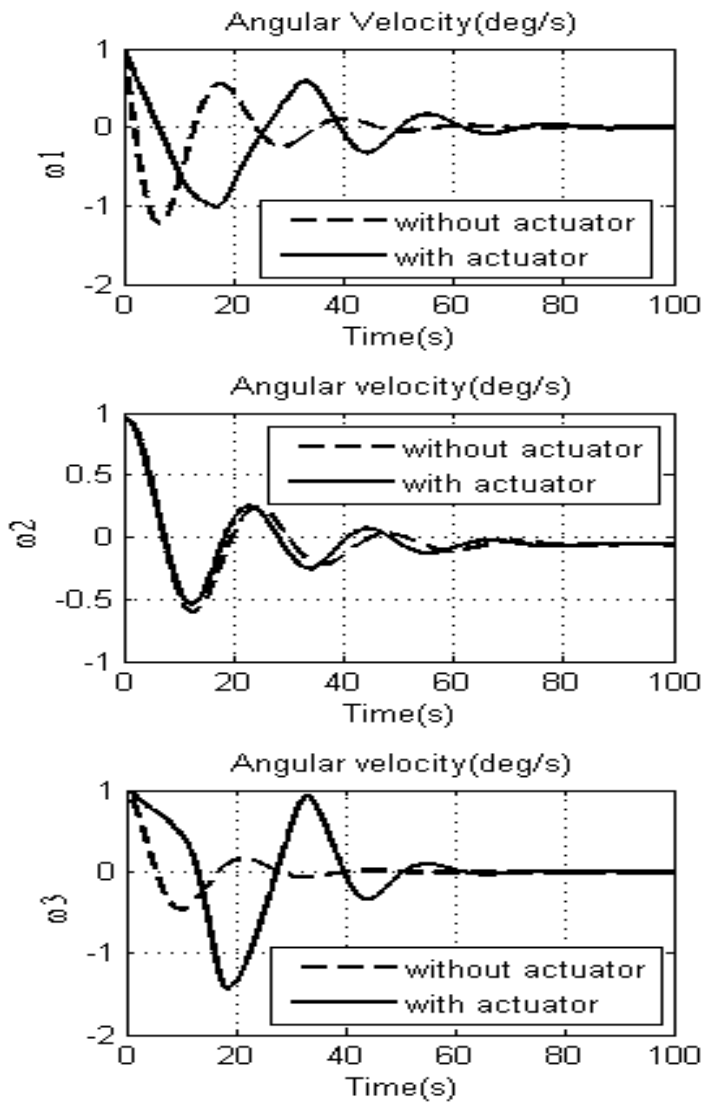

Fig 5: Angular velocities of rigid body
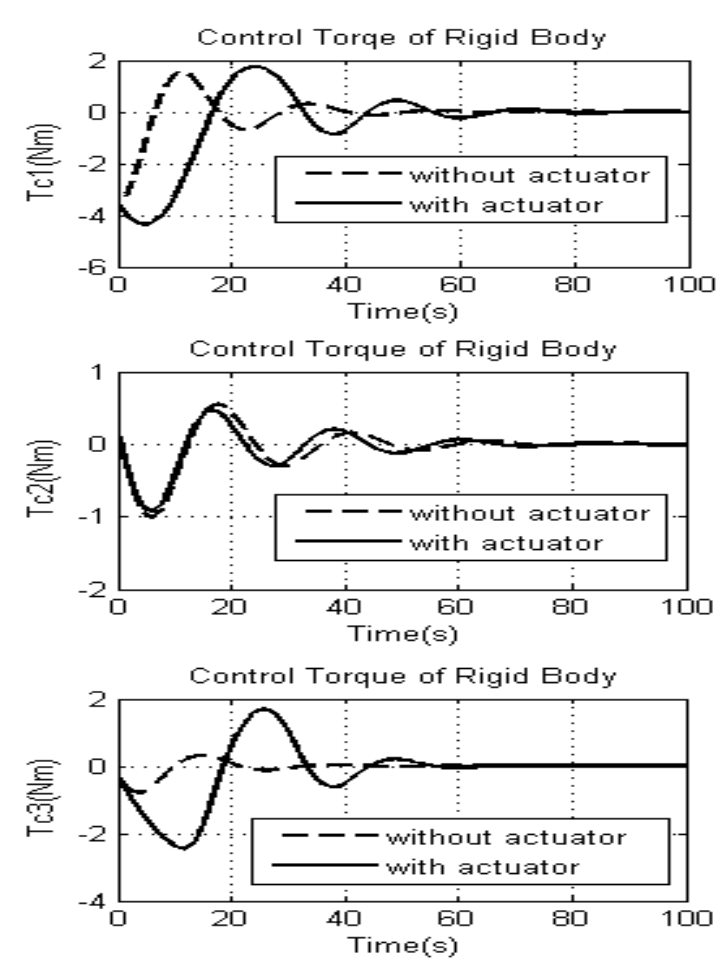

Fig 6: Required control torque of rigid body 

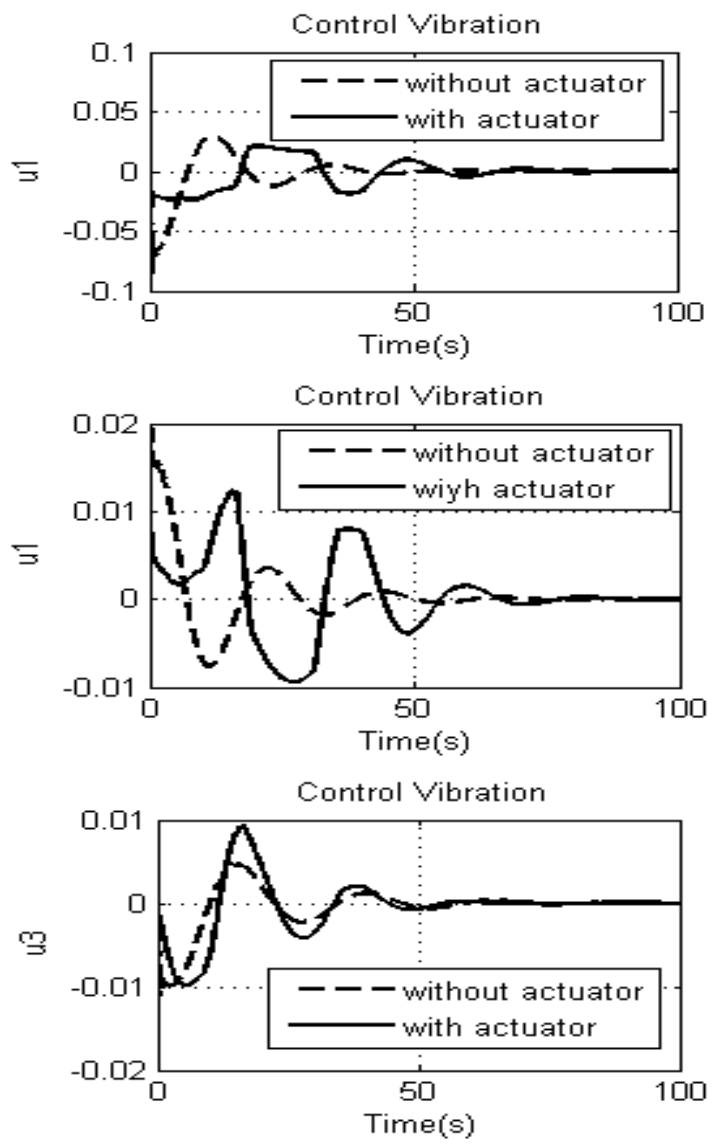

Fig 7: Control commands of flexible part
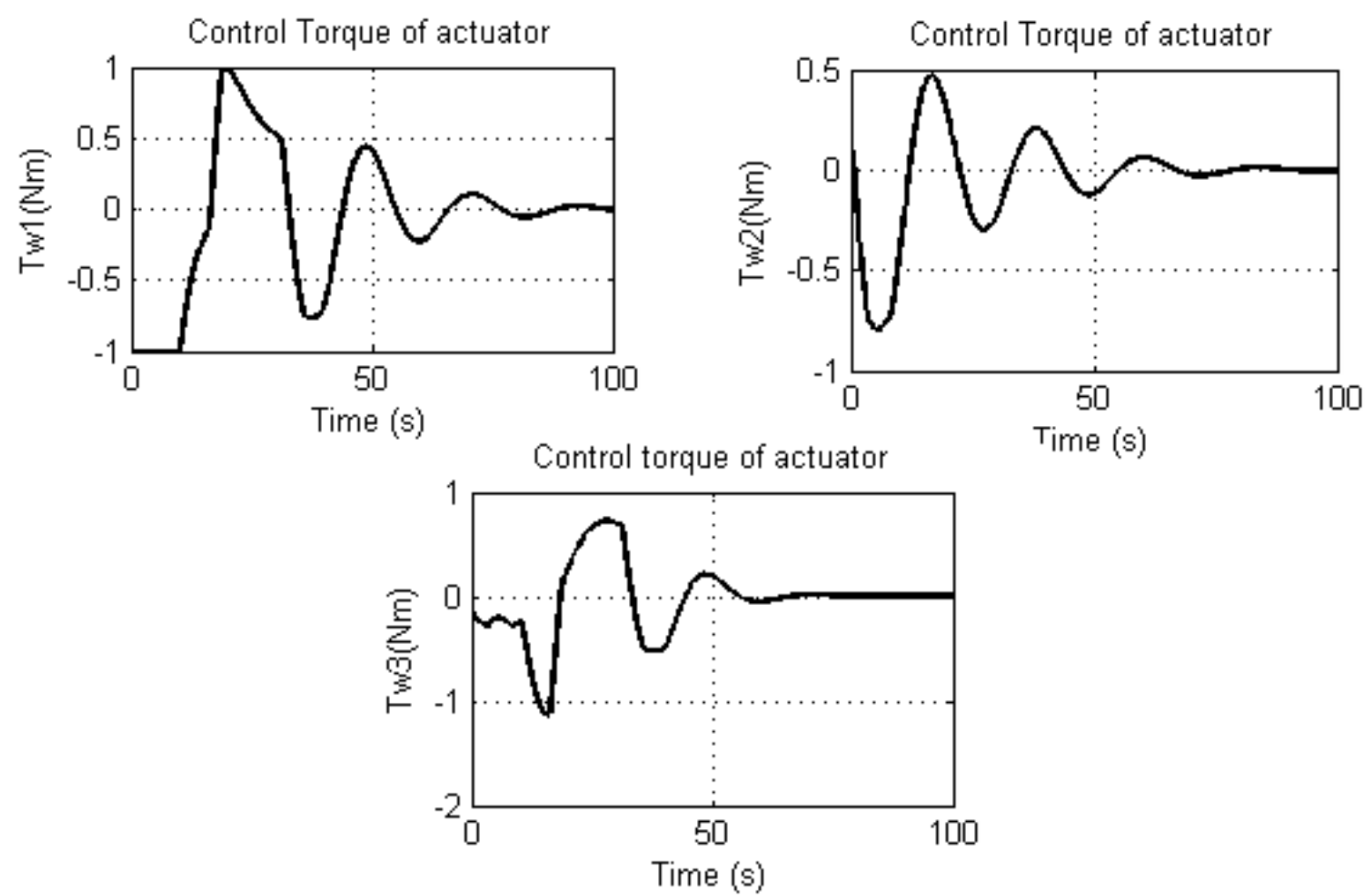

Fig 8: Control torques of reaction wheels with momentum management 

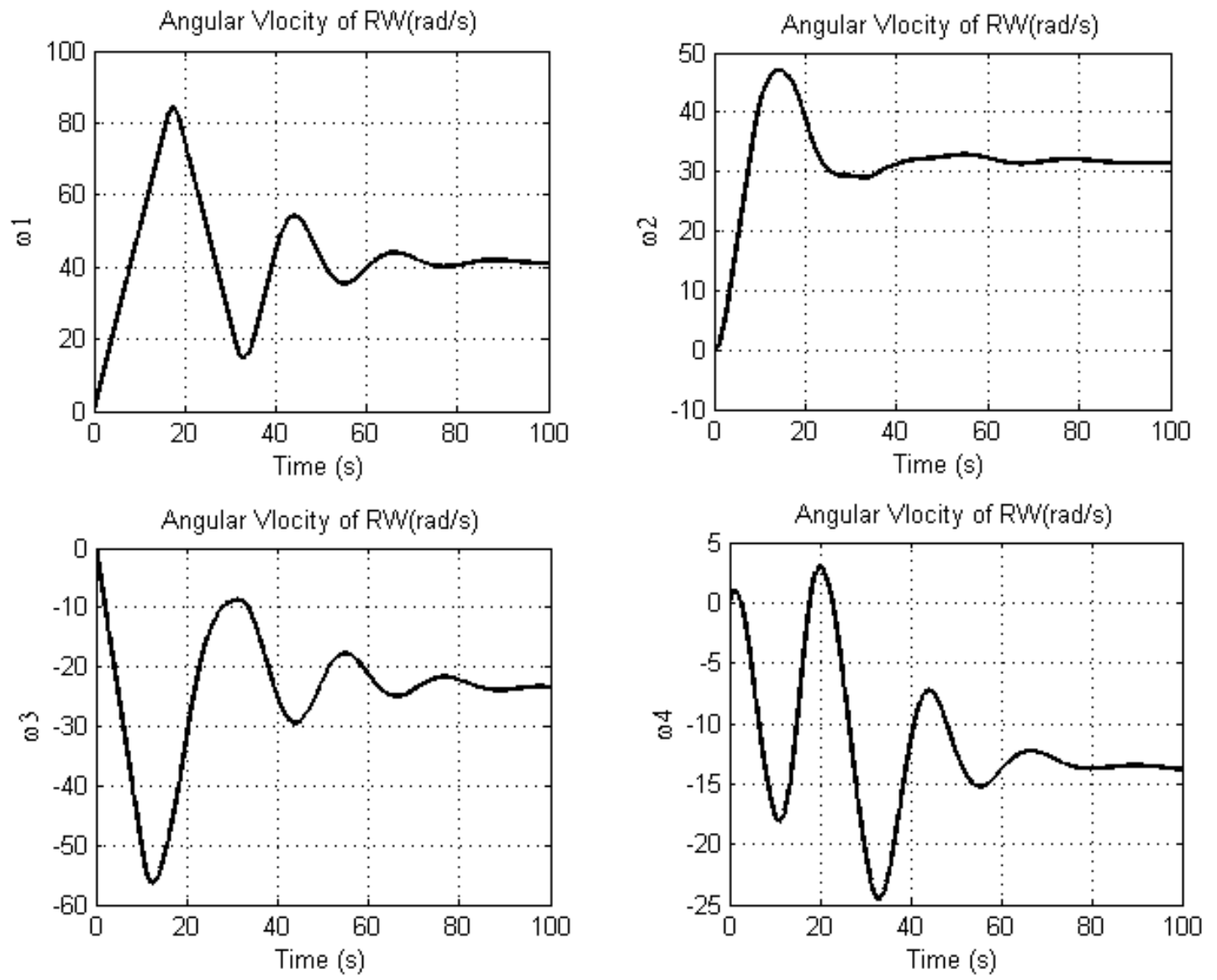

Fig 9: angular velocities of four wheels with momentum management

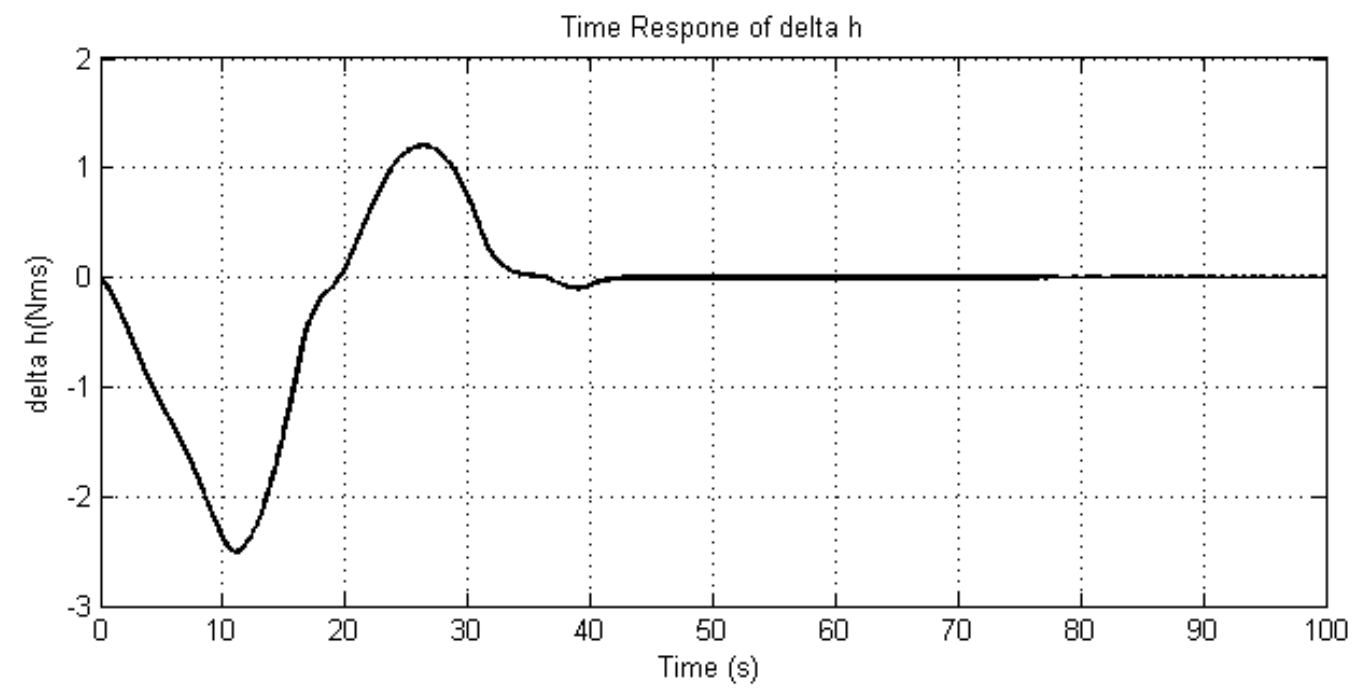

Fig 10: time response of angular momentum of four wheels with momentum managemen

\section{CONCLUSIONS}

In this paper a flexible satellite includes flexible appendages contacted to a rigid central body in a circular orbit has been considered. The control command law using the quaternion error vector for three axis control of rigid main body has been considered. The control gain coefficients have been chosen so that the satellite doesn't need to higher torque actuators (such as gyro). In this case the attitude control actuators are four reaction wheels. Therefore, the efficiency and reliability due to the use of four wheels, in the event of damage to one of the wheels. To avoid of reaction wheels saturation, momentum management and minimization of the momentum have been employed. Also the sliding mode control for damping vibrations of flexible body has been designed. The simulation results show that appropriate performance of controllers in presence of uncertainly and external disturbance. Finally the performance of control system between presence and absence of reaction wheels has been compared. As can be seen, vibration damping of flexible appendages is one of the most important challenges of attitude control of flexible satellite. 
Notch filter adaptive control can be added to control system. Also, it can use piezoelectric as active vibration suppuration actuator on the appendages.

\section{REFERENCES}

[1] Singh, Sahjendra N., and Rong Zhang. "Adaptive output feedback control of satellite with flexible appendages by modeling error compensation." Acta Astronautica 54.4 (2004): 229-243.

[2] Zhou, Wenya, et al. "High Accuracy Attitude Control System Design for Satellite with Flexible Appendages." Mathematical Problems in Engineering 2014 (2014).

[3] Bang, Hyochoong, Min-Jea Tahk, and Hyung-Don Choi. "Large angle attitude control of satellite with actuator saturation." Control engineering practice 11.9 (2003): 989-997.

[4] Wang, Qiyu, Jianping Yuan, and Zhanxia Zhu. "The application of error quaternion and PID control method in Earth observation satellite's attitude control system." Systems and Control in Aerospace and Astronautics, 2006. ISSCAA 2006. 1st International Symposium on. IEEE, 2006.

[5] Boulet, Benoit, et al. "Uncertainty modeling and experiments in \&Hscr; $\infty$ control of large flexible space structure." Control Systems Technology, IEEE Transactions on 5.5 (1997): 504-519.

[6] Guan, Ping, Xiang-Jie Liu, and Ji-Zhen Liu. "Adaptive fuzzy sliding mode control for flexible satellite."
Engineering Applications of Artificial Intelligence 18.4 (2005): 451-459.

[7] Ma, Yajie, et al. "Uncertainty decomposition-based faulttolerant adaptive control of flexible satellite." Aerospace and Electronic Systems, IEEE Transactions on 51.2 (2015): 1053-1068

[8] Slotine, jean , -jacques E . Applied nonlinear control. Prentice, 1991.

[9] Wertz, James R., ed. Satellite attitude determination and control. Vol. 73. Springer Science \& Business Media, 2012

[10] Shuster, Malcolm D. "A survey of attitude representations." Navigation 8.9 (1993): 439-517

[11] Gennaro, S. Di. "Output stabilization of flexible satellite with active vibration suppression." Aerospace and Electronic Systems, IEEE Transactions on 39.3 (2003): 747-759.

[12] Sidi, Marcel J. Satellite dynamics and control: a practical engineering approach. Vol. 7. Cambridge university press, 1997.

[13] Qian, Dianwei, Jianqiang Yi, and Dongbin Zhao. "Hierarchical sliding mode control for a class of SIMO under-actuated systems." Control and Cybernetics 37.1 (2008): 159. 